\title{
Design decisions in a Pascal-based operant conditioning system
}

\author{
HOWARD L. KAPLAN \\ Addiction Research Foundation of Ontario, Toronto, Ontario, Canada
}

\begin{abstract}
FREDL, a Pascal-based system for control of operant experiments, has been implemented on the IBM PC computer. In the foreground, recurring operations, such as decrementing counters that serve as local clocks, are controlled by a dictionary specifying automatic properties of userdefined variables, and random logic is implemented by a user-written Pascal overlay. In the background, the remainder of the program controls communication with the user, output of data to disk, and production of cumulative records.
\end{abstract}

There are two traditional approaches to computer control of operant conditioning experiments. In one approach, a special-purpose language that includes concepts specifically useful in these experiments is developed; several such implementations use state notation (Snapper, Kadden, \& Inglis, 1982). In the other approach, a generalpurpose language such as BASIC is used, and the same operating environment and vocabulary of functions designed for arithmetic and data processing are applied to real-time control. Recently, a third alternative has become available, that of systems with several built-in schedules and with experimenter control of the parameters and sequencing of the schedules, but no provision for creation of novel schedules; an example of this approach is OPN (Emmett-Oglesby, Spencer, \& Arnoult, 1982; Spencer \& Emmett-Oglesby, 1985).

At the Addiction Research Foundation, none of these alternatives would meet our needs in a cost-effective manner. Our primary interest is in schedules that lead to animal self-administration of drugs (including alcohol), with a possible secondary interest in the effects of such drugs on performance variables such as response times. Since drugs are not natural reinforcers for animals, complex schedules must be used to induce self-administration. Because we could not be sure what kinds of schedules would be developed in future years, a system such as OPN was ruled out on the basis of insufficient versatility. Although there was no doubt that state-diagram notation had the required versatility, we were concerned about the cost of software and interfacing of the newer implementations, and about the level of support for some inexpensive older implementations. Although the software pur-

This paper was presented in part at the annual meeting of the Society for Computers in Psychology, San Antonio, TX, November 7, 1984. The participation of Wan-Chung Cheng in the design of the hardware interface described here and the suggestions concerning this manuscript made by Bill Corrigall, Jack Orr, and Walter Schneider are greatly appreciated. The author's mailing address is: Addiction Research Foundation of Ontario, 33 Russell Street, Toronto, Ontario, Canada M5S 2S1. chase costs for using general-purpose languages would undoubtedly be less than those for some special-purpose operant systems, there were still two major questions: Would the language concepts facilitate a convenient notation for the operations to be described, and would the speed obtainable from such systems allow the costeffective control of multiple boxes? The notation question is important because many experimenters are already familiar with state notation, and any alternative notation must have clear advantages in order to justify abandoning a familiar way of working. The speed question arises because many high-level languages currently in use are interpreted languages (such as most implementations of BASIC) or semi-interpreted languages (such as many implementations of Pascal).

We ultimately decided to implement a novel operant conditioning environment, largely because doing so offered an opportunity to include new features not otherwise available at a reasonable cost. These features include the use of Pascal as an alternative to state notation, continuous screen display of selected experimental variables, two kinds of built-in simulation for testing, disk logging of responses at any desired level of detail down to the individual response, concurrent production of cumulative records on a general-purpose graphics printer, and use of a relatively inexpensive control bus for communication with the individual boxes. This system runs on an IBM PC enhanced with a relatively inexpensive, homebuilt, real-time interface. The system has been named FREDL, which stands for "Fairly Rational Experiment Description Language.' In presenting this history and current status of FREDL, I hope to illustrate how certain design decisions simplified the implementation, reduced the cost, speeded the development process, or allowed the computer to process more complex schedules.

\section{GENERAL SOFTWARE STRATEGY}

Real-time experiments in any discipline must be care- 
fully designed to ensure that critical events occur at the right moments. For programmers who are not limited to the operations explicit in a high-level language, and who can write assembler when necessary to provide critical functions (such as interrupt handling), the speed problem in operant conditioning experiments can be simplified by dividing the control program into two parts, an interruptdriven one to communicate with the boxes and a slower part, written in a high-level language, to communicate with the experimenter. This multiprogramming philosophy was discussed in more detail in an earlier article (Kaplan, 1980), in which I considered the possibility of sharing control of an operant experiment between assembler- and BASIC-coded sections.

It was my original intention to develop FREDL much along those projected lines: write a foreground interrupthandler in assembler, let it pass messages back and forth in a shared memory area, and let a background BASIC program interpret those messages for functions such as updating a screen display, writing data to disk, and producing graphic records. In the first stages of the project, the parts of the code that describe any one particular schedule would be written in assembler, but later a simple compiler would be developed to allow that code to be written by a less skilled programmer. Although the higher-level language chosen for the project was Pascal rather than BASIC, and although the particular schedules themselves are also coded in Pascal rather than assembler, the essential foreground-background philosophy was retained in the final design.

The structure of FREDL can be described as that of an operating subsystem (Kaplan, 1982) or support environment for operant experiments. Running under the standard PC-DOS operating system, it provides additional services for program modules that describe particular experiments. About one-tenth of the memory allocated to code is reserved for these modules, and the remaining code (about $60 \mathrm{~K}$, including the Pascal implementation's fixed run-time overhead) remains constant from experiment to experiment. This code includes a small number of subroutines that the experiment modules can call as needed: make random selections, save data on disk, decrement a counter and set a flag if the counter reaches zero, etc. However, the support environment is much more than a subroutine library, because it provides a large number of implicit services to complement the explicit ones requested via subroutine calls. To take a simple example, many real-time systems include a clock that is continually updated, so that programs simply may read the clock value whenever it is required. In an operant conditioning system, specific variables can be declared to be clocks, that is, to be automatically incremented or decremented in synchrony with the overall system clock. If the lists of such variables are stored in tables, then systemlevel code, which is often designed to execute with more efficiency than user-level code can achieve, can quickly update all such user clocks on a periodic basis.
Another way of creating automatic events is to code subroutines with deliberate side effects on their parameters or on system variables. For example, there may be a table associating the state of a particular program variable with an output device, such that whenever the variable is nonzero, the associated output bit is set. If all changes of state of that variable are accomplished using subroutines rather than using simple assignment statements, then the subroutines can monitor for changes to and from the 0 value and set the appropriate output bit accordingly. For example, if FoodHopper is a variable that is decremented on every tick of the system clock and is associated with the interface food presentation bit, then the programmer does not code

$$
\text { FoodHopper.value: }=15
$$

to turn on the hopper for $1.5 \mathrm{sec}$, but codes

$$
\text { setvalue(FoodHopper, 15) }
$$

instead. The setvalue routine implements the assignment statement, but also detects that the new value is nonzero, and therefore sets that box's bit of the associated output to active. Similarly, instead of coding

$$
\begin{aligned}
& \text { if } \text { (PressesToGo.value }>0 \text { ) then } \\
& \text { PressesToGo.value: }=\text { PressesToGo.value }-1
\end{aligned}
$$

the programmer can code

$$
\text { decrement(PressesToGo) }
$$

instead. The decrement procedure not only does the conditional subtraction of 1 , it also sets an associated variable PressesToGo.becamezero to true, for use in subsequent logical tests.

Taken together, these two ideas suggest that some of the work of coding an experiment should be accomplished by declarations rather than by executable code. For example, here are a few lines of pseudocode adapted from the declaration section of an early design sketch of the FREDL language:

\footnotetext{
Bit 0 is output, activates cage light, inactive is off, tied to session-timer, decrements every minute, initialized to 30 .

Bit 1 is output, activates timeout light, active is off, tied to timeout-timer, decrements every clock-tick, initialized to 0 .

Barpress-count is a counter, initialized to 0 .
}

The first sentence describes a bit that stays on for $30 \mathrm{~min}$; the clock named session-timer is initialized to 30 when the session starts, and moves toward zero at the rate of one count per minute. The second sentence describes a bit that is normally on, but is turned off for a specific amount of time under program request, accomplished by setting the variable timeout-timer to the number of clock ticks for which the time out is to last. The third sentence describes a strictly internal counter, tied to no input or output bit. Attributes similar to the ones in this pseudocode remain a property of the software counters included in the FREDL implementation. 
In FREDL, the variables that retain information about any box between 100 -msec clock ticks are known as "counters." These counters are not simply arrays of variables (one per box), with names such as "Reinforcements" (the total count of reinforcements delivered). Instead, they are arrays of associated sets of variables (what Pascal calls "records"), whose updating generates automatic side effects, such as setting an associated output bit to "on" whenever the variable is nonzero. For example, one kind of object is a TickCounter. Every $100 \mathrm{msec}$, its value is inspected, and if it is positive, the value is decremented. The counter can have an associated output bit, which is set to its nonresting state whenever the counter is nonzero. In addition, there is an associated flag that indicates when the counter has just become zero. Therefore, to activate a food hopper for $1.5 \mathrm{sec}$, the programmer simply invokes the procedure to set the associated TickCounter to 15 , and the remainder of the program automatically activates the hopper, decrements the counter 15 times, and deactivates the hopper. Another kind of object is an InputRecorder, which is incremented on every barpress and which also sets a flag in the 100 -msec interval following such a barpress. A third kind of object is a Parameter, which has a value that can be changed by the experimenter under keyboard control before or during a session. Using such a Parameter, the experimenter can code a VR schedule with an (arbitrary) value of $\mathbf{2 0}$ responses per reinforcement, and then change that value to the appropriate value for each box at the time the experiment is run.

Although the facilities within FREDL are more general than those of state notation, they do include the latter's most important properties as subsets. A ManualCounter maintains its numeric value until changed by an explicit program statement. Therefore, it has the properties required of a state for paradigms most naturally described by a kind of state notation, and it is possible to give such a counter a name such as "FirstParallelState" to emphasize such a use. In the code describing an experiment, case statements based on the value of such a counter can select blocks of code analogous to state-transition rules in state notation. Communication between parallel state systems can be accomplished using TickCounters, and such counters even provide a facility for delayed " $Z$ " pulses.

The definitions of the counters are collected by an interactive dictionary-preparation program. A screen display allows the programmer to define variables and their attributes by filling in a CRT form that prompts for appropriate values: a number for initialization, a state (normally off, normally on, or flashing when on) for outputs, etc. Each of the different types of definition-input, output, counter, randomization list, and cumulative recordis updated in a separate, independently scrolled screen window, so that the programmer can easily monitor the relationships between the definitions. Other properties of these software objects that can be defined are which screens should display their current values (any one screen is limited to 16 counters, but there can be up to 16 different screens) and whether their final values should be printed after the cumulative records are complete.

The dictionary-preparation program writes the definitions to two different disk files, one containing the properties, initial values, etc., in the internal format to be used while the program runs, and the second containing the same information in the form of variable names and comments that are included in the Pascal source of the experiment. For one specific paradigm, discriminated fixed interval (FI) with a flashing light to indicate the expiration of the interval, Listing 1 shows an example of the contents of this latter file, and Listing 2 shows the code that describes the actual paradigm logic. Note that the code does not include any subscript or other indication of which box is being processed; the Pascal language allows the specification of that information using a "with" statement that applies to an entire block of code such as this one. The module is compiled as a discarded main program and an experiment-specific overlay, and the overlay is eventually executed by the run-time environment.

To reduce peak run-time processing requirements, all random selections other than simple selections of a random number from 0 to $n$ are performed with the use of precompiled lists of choices. For example, to implement an approximate random ratio schedule (probability of reinforcement independent of the number of preceding unreinforced trials), a distribution of up to 100 interreinforcement intervals is generated with appropriate mean and maximum values. Such lists can then be sampled either with or without replacement; the former maximizes the uncertainty of each succeeding ratio, but the latter can be used to guarantee that the overall distribution achieves its nominal mean. Sampling without replacement is performed from an independent copy of the original list for each box, and an exhausted list is automatically set back to its full condition, allowing such sampling rules as "every 8 reinforcements are delivered after two each of $5,10,15$, and 20 responses, randomly ordered."

Of the software strategy described here, only the initial idea of having a separately compiled experiment module, activated on every interrupt whether or not there are disk operations in progress, preceded the beginning of development. That initial idea influenced the decision to implement the project on the IBM PC, and specific features of the languages available then led to further development decisions.

\section{THE DECISION TO USE THE IBM PC COMPUTER}

The IBM PC computer is becoming very common in laboratories for some good general reasons: It is widely available, it is not exorbitantly expensive, the hardware is reliable, there is a wide range of interface boards available, and there are many languages implemented upon it. The same, however, can also be said of computers such as the Apple II, the microprocessor versions of the PDP- 
Listing 1: Dictionary for a discriminated FI experiment

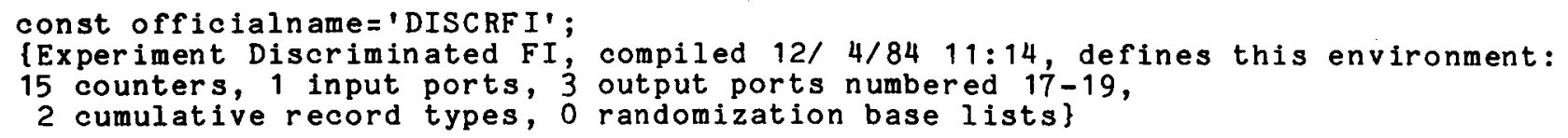

\{Port 1: OnlyBar, symbol 18, is an input responding to logic onset\}

\{Port 17: Houselight, symbol 15, is an output with active logic off\}

\{Port 18: Beeper, symbol 13, is an output with active logic off\}

\{Port 19: FoodHopper, symbol 2, is an output with active logic on

\{The following counters have been defined:\}

type counterlist =record

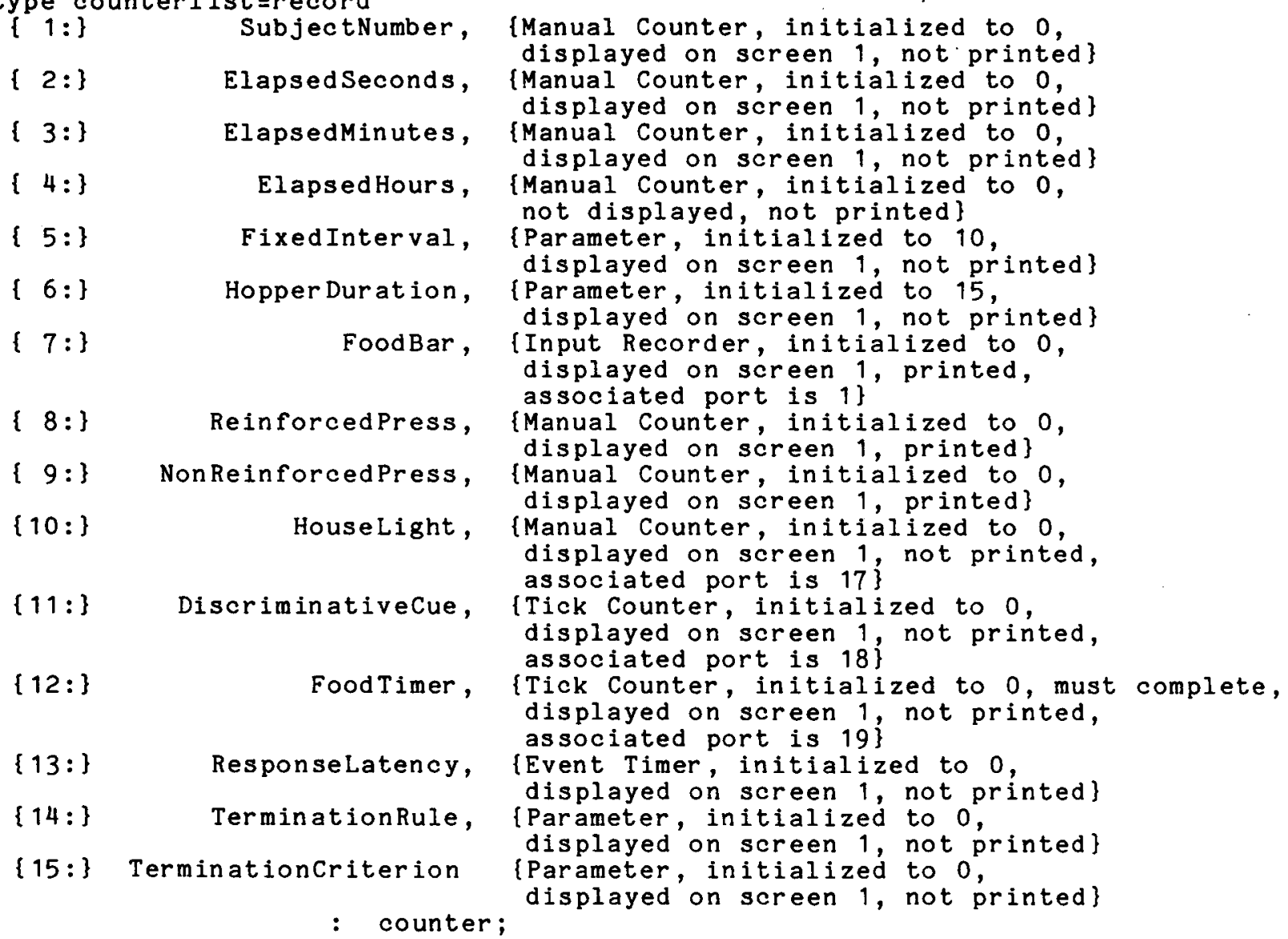

ICumrec 1: 1 seconds per pixel, with \{Cumrec $2:$ response 7 reinforced by 8 , annotated for each change on 5 , 6 \} response 8 , response 9 , annotated for each change on 5,6$\}$

Listing 1. Dictionary for the DISCRFI study. The interactive dictionary-preparation program produces the dictionary in two forms, a disk file of condensed definitions used in the run-time system and this form, text interpretation of the definitions for inclusion in the source of the Pascal code defining the paradigm.

11, and various 8-bit $\mathrm{CP} / \mathrm{M}$ systems, all of which are also found in many laboratories. However, there are some particular advantages of the IBM PC, especially compared with the 8-bit systems, that recommend its use for this kind of experiment. Most of these advantages arise from the suitability of the IBM PC and its PC-DOS operating system for a relatively simple multiprogramming system, one in which the programmer rather than the operating system resolves resource conflicts among the multiple programs. 


\section{Listing 2: Program logic for a discriminated FI experiment}

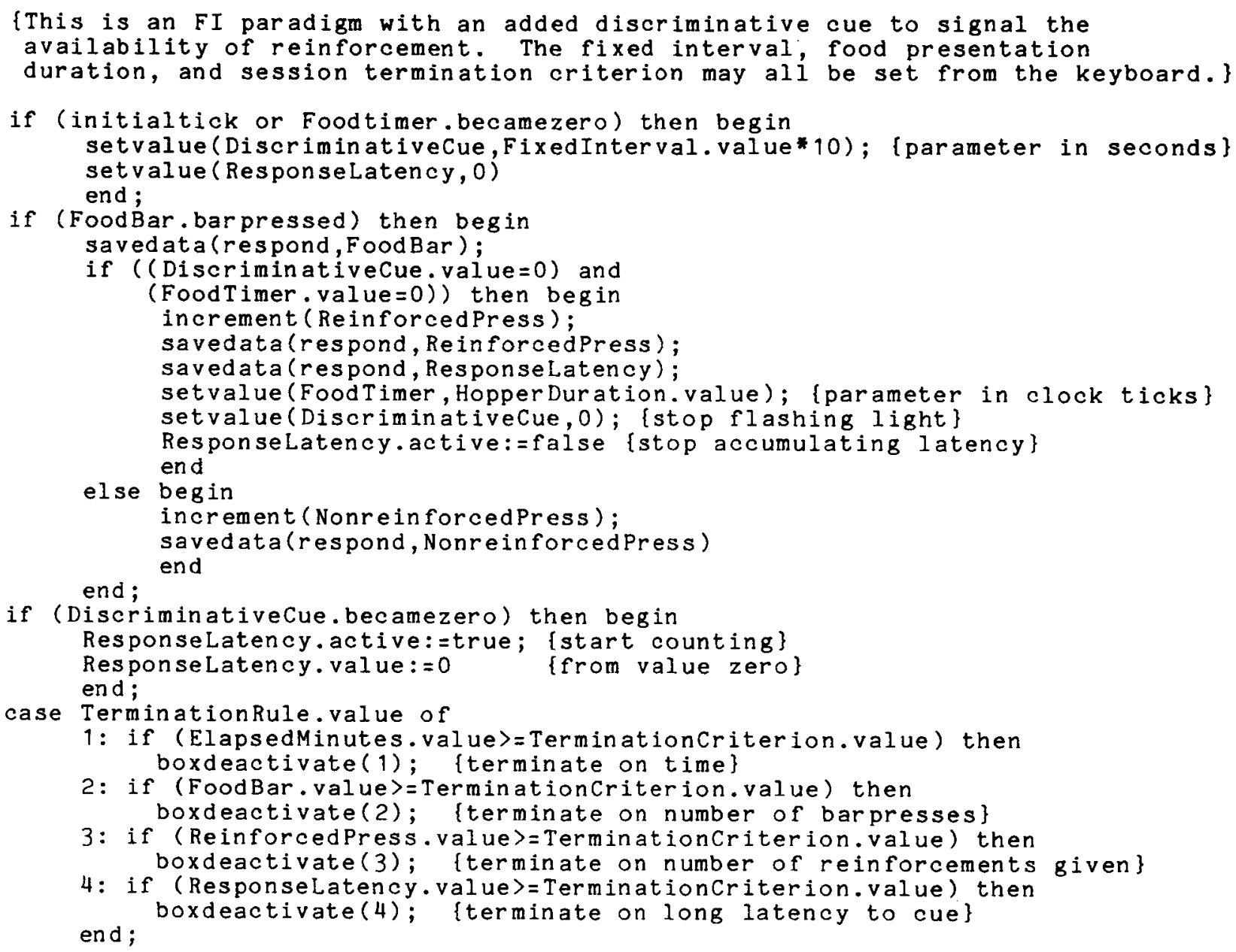

Listing 2. Source code for the DISCRFI study. The code refers to counters previously defined in the source file produced by the dictionarypreparation program, and consists of a sequence of begin-end and case structures, occasionally nested; there are no GOTOs. When the program refers to a counter in an assignment or comparison, it specifically refers to the arithmetit part (".value") or the boolean (true or false) part (".barpressed," “.becamezero," or “active"). However, some subroutine arguments refer to the counter as a whole, with the subroutine picking out the parts as needed and possibly referring to the dictionary in order to decide how to process them. If side effects (such as turning outputs on or off) are needed, then counters must be updated using procedures such as setvalue; otherwise, they may be updated with assignment statements.

The IBM PC is commonly available with $256 \mathrm{~K}$ of memory, and larger configurations are possible. This much memory has some valid uses in a multiprogrammed system. First, it provides the space in which the multiple programs can reside, including a foreground partition large enough for several paradigms to be coded simultaneously. Second, the generous amount of memory allows for large buffers, memory partitions that hold a back$\log$ of information awaiting transfer to or from diskette. As events that are important enough to be logged to diskette occur, the foreground program does not actually log them; instead, it leaves copies of the intended disk records in a buffer where the background program eventually finds and outputs them. This division of tasks al- lows the background program to wait for disk operations without delaying the foreground, but requires enough memory to hold the peak number of events that the foreground can generate between background disk-output operations. Third, a large amount of memory allows control tables to be stored in a format optimized for speed rather than for conservation of space.

A second important feature of the IBM PC is that diskette transfers occur on a DMA (Direct Memory Access) basis. Although the CPU is used to initiate disk operations, individual data bytes are moved without CPU intervention, and disk operations can be interrupted at any time without loss of data integrity. This feature is critical for an operant system such as this one, in which the fore- 
ground program must process events every $100 \mathrm{msec}$ whether or not there is a disk operation in progress.

Almost all interaction with the standard PC-DOS operating system is performed by the background program. The foreground program, which processes the interrupts, accesses the screen only by directly POKEing screen memory, not by using any of the standard system calls. The box-controlling I/O ports manipulated by the interrupt handler are all unknown to PC-DOS, rather than being controlled through user-supplied DOS device drivers. These restrictions are necessary because PC-DOS is a single-user operating system that assumes that no userlevel system calls will interrupt other user-level system calls referring to the same devices. In order to satisfy this requirement, the operant system foreground rarely interacts directly with PC-DOS; most operations are mediated through the background program. The only exception is that all reads from the keyboard are performed by the foreground, so that it can intercept characters intended as emulated barpresses in one of the two test modes; the characters not recognized as such emulated presses are left in a buffer for the background to interpret as operator communication.

The hardware environment required for FREDL is straightforward and not exorbitantly expensive. The standard components required are an IBM PC (or any reasonably compatible equivalent) with $256 \mathrm{~K}$ of memory, two diskette drives, the color/graphics display card, an RGB or composite-input color or monochrome monitor compatible with the output of the display card, a parallel printer port, and an Epson FX-80, FX-100, or equivalent printer. For the operant interface, we started with a board from John Bell Engineering, costing $\$ 300$ and including three 8255 interface chips (we use one of the three), low-speed analog I/O (which we do not use), a $32767-\mathrm{Hz}$ clock (which we divide by 3277 for $100-\mathrm{msec}$ interrupts), and interrupt request logic. The three 8-bit ports from the 8255 are used to control a 23-wire bus servicing eight boxes, with logic power, line drivers, and connectors for the bus located in a separate chassis external to the PC.

\section{THE DECISION TO TRANSPOSE THE USUAL BUS ORIENTATION}

On common 8-bit computers using the $6502 \mathrm{CPU}$, there are VIA or PIA interfaces in which individual bits of a single interface byte can be set to either input or output. However, computers using the 8086 or $8088 \mathrm{CPU}$ more typically use the Intel 8255 interface chip, in which two of the three interface bytes must be set to each be entirely input or output. This suggests transposing the usual kind of logic in which a single interface byte or word is associated with a box and the individual bits indicate actions within that box. Instead, our interface lets the bits of an I/O byte correspond to eight different boxes, with corresponding functions all input or output simultaneously. This simplifies both the interfacing and the programming. Interfacing is simplified because each box needs to inter- face with only two data wires (one each for input and output bits) in addition to two clocking bits and logic ground. One clocking bit latches outputs into a shift register or clocks inputs from a seçond register; the other clocking bit initiates transfer of all output bits from the shift register to device latches, and the trailing edge of that clock signal transfers input latches to the input shift register.

This design not only keeps wiring and interfacing costs low, but also simplifies the software, because corresponding operations on different boxes' data (such as software detection of transitions from bar-off to bar-on states) can be done in parallel using bytewise logical operations. For example, if variable oldbyte holds the previous state of an input byte and newbyte contains its current state, then the expression ((not oldbyte) and newbyte) indicates which bits became 1 on the current clock tick; in other words, the expression identifies bit onsets. This parallel processing contributes to the overall system speed.

To simplify the interfacing, each input event can be either debounced and used to trigger a one-shot of about 150 -msec duration (to ensure that it is sampled on at least one 100-msec clock tick) or else used to set a latch that will be cleared after the bit has been read. In the former case, software logic as described above prevents the second and subsequent detections of the same barpress from being interpreted as new events. The software also allows either the high or low logic level to be used as indicating the event of interest. Outputs can be specified as having active states of off, on, or oscillating. All outputs are off when their associated boxes are not activated. During normal operations, some outputs (such as houselights) are normally on, but can be turned off for events such as time outs; others, such as food or drug dispensers, are normally off but are briefly activated under program control. Oscillators with a fixed duty cycle of $50 \%$ can be specified to have any half-period from 1 to 10 clock ticks, and their resting state is off.

\section{THE DECISION TO REJECT BASIC AND ASSEMBLER}

The original system design for FREDL was that only the most critical section of code, the foreground interrupt handler, would be coded in a high-execution-speed language, and the rest could proceed at the rate provided by BASIC. During development, the interactive BASIC environment would be a great convenience, and subsequently the code could be compiled for run-time efficiency. In addition, among the languages available on the IBM PC and possessing a reputation for being relatively free of errors, only the IBM implementation of BASIC provided the graphics functions necessary for screen display of cumulative records. However, the abandonment of three incorrect ideas-that no compiler would provide enough speed for the foreground program, that BASIC provides a good program development environment, and that screen display of cumulative records is desirableled to the ultimate decision to use a different language environment, Turbo Pascal. 
In some ways, the program-development environment provided by BASIC is excellent. There are no separate compilation or link stages, partial programs can be tested easily, and the presence of source in memory allows the flow of control to be traced at the source statement level during debugging. In addition, the language is familiar to many programmers, so that future system maintenance would not require specialized skills. On the other hand, the vocabulary of control structures available in IBM BASIC is very limited, better than that in FORTRAN IV but lacking multiple-line, nested IF-THEN-ELSE control. This lack means that BASIC programs tend to require many implicit and explicit GOTO statements, features that tend to make program understanding and development more difficult than in better-structured languages. (The argument that GOTOs provide for a relatively poor control structure is one argument against the use of state notation. States can be viewed as blocks of conditional statements, with the transition from state to state a kind of computed GOTO indicating which block of statements to process on the next clock tick.)

Other languages have much wider varieties of data structures than the elements and arrays provided by BASIC. To provide a very simple example, let us consider a table associating a list of keywords with numeric values. In FORTRAN or BASIC, this would be represented as two parallel arrays, one of character strings and a second one of numbers, both having the same dimension or range of valid subscripts. Subsequent operations on the array, such as inserting a new association, would require separate, parallel operations on the list of strings and the list of values. In contrast, languages such as PL/1 and Pascal allow the definition of structures in which each component is an associated pair of string plus number, so that the string, the number, or the pair as a whole can all be referred to in subsequent operations. Another advantage of having an array of such structures rather than parallel arrays of the component parts is that the differenttype elements of any one structure occupy contiguous memory locations, allowing relatively rapid storage and retrieval of such structures on diskette. In FREDL, for example, the dictionary, the counters, and the event data records are all structures consisting of several components of different types.

A further deficiency of BASIC is that there is no control over the scope of variable names. It is the responsibility of the programmer to ensure that the variable "I," used as a loop index in one routine, does not conflict with the use of that variable in another routine, because both refer to the same memory location. This makes it very difficult to safely combine independently coded routines without generating a set of long but guaranteed-unique variable names such as "I.WITHIN.ROUTINE1".

Few of these deficiences of BASIC, which are generally well-known, are important in short programs, those whose structure an average programmer can easily keep in mind while working. However, as it ultimately developed (and would not have developed given the memory limitations and other constraints of the BASIC environ- ment), FREDL occupies about 60 pages of source code in its current version. This is clearly of a magnitude requiring a language designed on the assumption that large programs will be coded in it.

Many, but not all, of the disadvantages of coding in BASIC apply to assembler. Since the foreground program was intended to be relatively short (its Pascal implementation takes only seven pages), details such as preventing conflicts among uses of the same variable could be left to the programmer rather than to the compiler. On the other hand, the development environment for assembler commonly used on the IBM PC requires separate editing, compilation, linking, and debugging stages, and any alternative providing adequate speed in a more convenient environment is certainly attractive.

\section{THE DECISION TO USE PASCAL}

By a process that was as much a matter of fortunate accident as of rational investigation, I was investigating the Borland International implementation of Pascal, called Turbo Pascal, at the time that I was working on the early stages of FREDL. This was my first experience with Pascal, and as I gained a personal appreciation of how the language facilitated my work as a programmer, I started to understand the limitations of BASIC, which are summarized in the previous section. This compiler was originally selected largely on the basis of object code speed (it is not an optimizing compiler, but it produces native code of reasonable efficiency) and a comfortable programmer's environment (memory-resident editor, compiler, source code, and object code, at least for small modules). I only later realized that the Pascal structured-programming environment was important for developing both system code (the permanent facilities available to all experiments) and user code (the description of specific experiments). Although, by a rough estimate, the efficiency of the code produced by Turbo Pascal is only $25 \%$ of that of assembler code, the idea of coding specific experiments in assembler was abandoned, because the speed of the Pascal implementation made it possible to code both the background (communication with operator, storage on disk, etc.) and foreground (control of boxes on a strictly timed basis) portions of the program in Pascal.

For this strategy to work, it is essential that the code produced by the compiler be native code rather than $\mathbf{P}$ code or some other interpreted code, regardless of how fast the interpreter is. If both the background program and foreground interrupt handler are written in Pascal that produces native code, then the only values that need to be saved and restored upon interrupt are the $\mathbf{8 0 8 8}$ machine registers. On the other hand, if both levels of program use a common interpreter, then memory may contain additional values (such as software program counters, memory pointers, etc.) that would need to be saved and restored upon interrupt. By similar logic, it is important that any functions and procedures shared by the foreground and background are fully reentrant, that is, do not 
write into any memory other than their parameters and the stack.

To implement FREDL, it was necessary to write a small portion of the system, less than 200 bytes of object code (compared with about 50,000 bytes produced by the compiler), in machine language. This machine language code primarily implements saving and restoring registers in interrupt handling, but also interfaces the code to a few operating-system facilities for which the interface would otherwise be too time-consuming. Should future experiments require a more efficient implementation, there are many places in which the foreground code, especially some small, frequently called subroutines, could be speeded by recoding parts in machine language.

Because Turbo Pascal is a relatively efficient programming language, it is practical to describe FREDL experiments with a mixture of code and tables, rather than having them be entirely interpreted from tables, as might result from implementing state notation. Tables, like lines of BASIC, need to be interpreted: Code must inspect the items in the tables, compare those items with constants or other table items, and execute logic based on the results of those comparisons. This is similar to what a computer CPU does when it fetches items from the special table of numbers known as a machine language program, except that a CPU is generally much more efficient at this than is any programmer's table-interpretation code: This is why compiled programs, which generate native code, are generally much faster than interpreters are. The strategy of dividing the program decisions into recurring ones, such as the regular decrementing of TickCounters stored in tables, and random ones, such as distinguish among operant paradigms, was therefore facilitated by two properties of the Pascal implementation: Its ability to handle much more complex data structures than can FORTRAN, BASIC, or assembler made table interpretation straightforward, and its execution efficiency made compilation of random logic equally straightforward.

Pascal notation also facilitates the control of side effects. Because variables can be embedded in structures and passed to subroutines within those structures, concise procedure calls can accomplish much. For example, the "decrement" procedure has one parameter, the name of a counter (with an implicit subscript of the box number). Since this counter is actually a structure with three components (the current arithmetic value, the current logical value which will be set to true if and only if the counter is decremented to zero, and a pointer to the dictionary table definition of the counter), the subroutine can easily use the fact that the counter reaches zero to update the output port image associated with that counter. Since a procedure call is implemented with just the procedure name, rather than with an explicit "call" verb, the syntax of procedure calls includes very natural phrases such as "increment(CorrectPresses)" rather than the more clumsy "call increment(CorrectPresses)."

Although Pascal is, for many purposes, a language more suitable than BASIC, it is much less familiar to many experimenters. Many of the features within Pascal, such as records, "while" and "repeat" constructions, and heap management, have no counterparts in familiar BASIC implementations. However, these differences should not prevent experimenters from using FREDL, because the subset of Pascal operations that can be safely executed within the foreground program includes only simple decisions (including case structures), begin-end blocks, assignments, and procedure invocations. Learning the Pascal syntax, punctuation, and concepts required for most straightforward paradigms should require only a few hours.

\section{THE DECISION TO USE FIXED-RATE INTERRUPTS}

There are two general strategies available to the experimenter implementing an event-driven system, either to interrupt the CPU on every significant event or else to interrupt it at a fixed but fast rate. Fixed-rate interrupts certainly allow for a simpler implementation: There is no need to write separate interrupt handlers for clock and other interrupts, and there is no need to worry about what happens when the clock handler interrupts the event handler or vice versa. The only real argument in favor of responding to events when they occur rather than when the next clock tick occurs is that it allows for a uniformly faster reponse by the computer to the event. However, this argument is not correct. If only one external interrupt occurs at a time, then the response will in fact occur more rapidly; however, if two such events occur nearly simultaneously, then the response to the second will be delayed while the first is processed. In other words, although the average response might be faster, the delay in processing the response becomes an uncontrolled function of what is occurring in other boxes at the same time. On the other hand, with a fixed-rate clock of sufficient frequency, the response time is a short interval with a distribution that is independent of other boxes' events. In the FREDL system, the clock rate is $100 \mathrm{msec}$. Any event will be detected with an average delay of $50 \mathrm{msec}$, bounded by 0 and $100 \mathrm{msec}$, and the computer's response will occur on the next clock tick 100 msec later. Providing that the program logic has been properly tested for its ability to execute within $100 \mathrm{msec}$ windows, these times are independent of system load.

Upon every 100-msec interrupt in FREDL, the following events take place:

(1) An internal decimal-format elapsed-time clock is updated, and the current time is rewritten to the screen by direct deposit of digits into screen memory. Time is displayed in HH:MM:SS.T format, with T representing tenths of a second. The time-consuming update of digits other than $\mathrm{T}$ occurs only on the first clock tick of each second, when $T$ is 0 and when the special processing mentioned in Sections 6 and 8 does not occur for any box.

(2) Unless a keyboard simulation is in progress, all outputs defined by the user are updated from byte images maintained in memory. If a keyboard emulation is in progress, then the outputs are interpreted as screen charac- 
ters instead, and are written directly to screen memory, so that the user can see which outputs are momentarily active.

(3) The DOS keyboard input buffer is scanned for any keypresses occurring since the last clock tick. All keypresses other than function keys 1 to 10 are passed to the background program for further interpretation. If a keyboard simulation is in progress, presses of function keys 1 to 10 are interpreted as simulated barpresses from the first five boxes, and the sampling of the interface in Section 4 is bypassed.

(4) All inputs defined by the user are sampled, and appropriate masks implement detection of on state, onset, off state, or offset independently for each type of input.

(5) The detections of barpresses from Section 3 or 4 are distributed among the active boxes' boolean variables of the form CounterName.barpressed.

(6) If the tenths-of-a-second digit of the elapsed time clock is 1 to 8 , then the status of the corresponding box ( 1 to 8 ) is inspected to see whether the box requires activation or deactivation, these being activities that take more CPU time than the usual every-interrupt activities. Limiting such special inspections to one box per interrupt reduces the peak load upon the CPU. Whenever a box is activated, the system elapsed time is written to the disk event buffer, and when the box is deactivated, the box's own elapsed time since activation is written to the buffer.

(7) For each box separately, all TickCounters are decremented and "active" ManualCounters are incremented.

(8) If the tenths digit of the internal clock matches the box number, then any SecondCounters are decremented. If, in the last second, the user has used the keyboard to change any parameters, then such changes are written to the disk buffer. On each 60 such decrements, the box's MinuteCounters are decremented. Again, the purpose of having such counters is to reduce the peak response demands on the CPU by having some lower-precision timing done on a round-robin basis.

(9) Continuing for each box separately, the specific user code for the box is executed. Disk records are generated only by specific request in the code, with the exception of choices of new random items from lists, where a disk record is automatically generated. Any changes to output bits caused as a result of this code are implemented at the next clock tick.

\section{DISK FILE MANAGEMENT}

In FREDL, there are six run-time file types associated with any experiment, all of which incorporate the eightletter experiment name as the first part of the name. Three of these are static (read-only) files: the experiment code overlay EXPNAME.000, the dictionary file EXPNAME.DIC, and an optional file of randomization lists, EXPNAME.RND. The other three are updated or created in every session, and maintain the permanent record of session events:

(1) Event data, stored in files of the form EXP-
NAME.nnn, where nnn is even and where each record represents one programmer-defined interesting event, such as a barpress, a reinforcement, or a change of experimenter-controlled parameter. Each such record includes the box number, the time of the event to the nearest second, the number of the counter being recorded (in the order in which they are named in the dictionary), the counter value, and a 1-byte offset to the next disk record for the same subject. When two records differ by more than 255 positions, dummy chain records are inserted as needed.

(2) Index data, stored in files of the form EXPNAME.nnn, where nnn is an odd number one greater than that of an event data file and where each record represents a summary of one subject's data and cumulative record pages already printed for one session.

(3) History data, stored in a single file of the form EXPNAME.HIS, where each record lists one session's subjects by number and box and indicates whether each subject's data are complete and are fully printed.

When FREDL is started, the file EXPNAME.HIS is examined, and the date and status of the last session recorded are reported. The operator then has the choice of completing the printing of any session's data, of reprinting any data, or of starting a new session. In the last case, the program automatically assigns the next available pair of even and odd numbers for the data and index files, reports the session number and file numbers to the operator, and opens the files. In the other two cases, the operator is asked for the session number that is to be reprocessed; if printing has not completed, it automatically resumes, and otherwise the operator is asked which graphs are to be reprinted.

\section{THE REAL-TIME OPERATOR INTERFACE}

In the time available between servicing interrupts, FREDL maintains a continuous screen display of selected counters' values; such a display is included in Figure 1. This display includes the total elapsed time for the session, separate elapsed times for each box, and the current values of up to 16 counters per box. In addition, the experimenter has the following vocabulary of commands that can be entered at any time, where uppercase indicates keywords typed exactly as shown, lowercase indicates other parameters, and text in brackets is not part of the command but is included here to help explain further how the commands work. While the program is running, the operator can type any command name followed by a question mark, and FREDL will reply with the proper syntax and argument list for that command.

\footnotetext{
ACTIVATE box-number WITH subject-number DEACTIVATE box-number/ALL

SET parameter-name box-number TO new-value

TURN ON/OFF bit-number IN [inactive] box-number/ALL

[continuously display counters belonging to] SCREEN screen-number

[display disk-recorded] EVENT eventnumber

[display] SUBJECT subject-number [box, eventnumber range, elapsed time]

[display] FINALVALUE counter-name FOR subject-number
} 


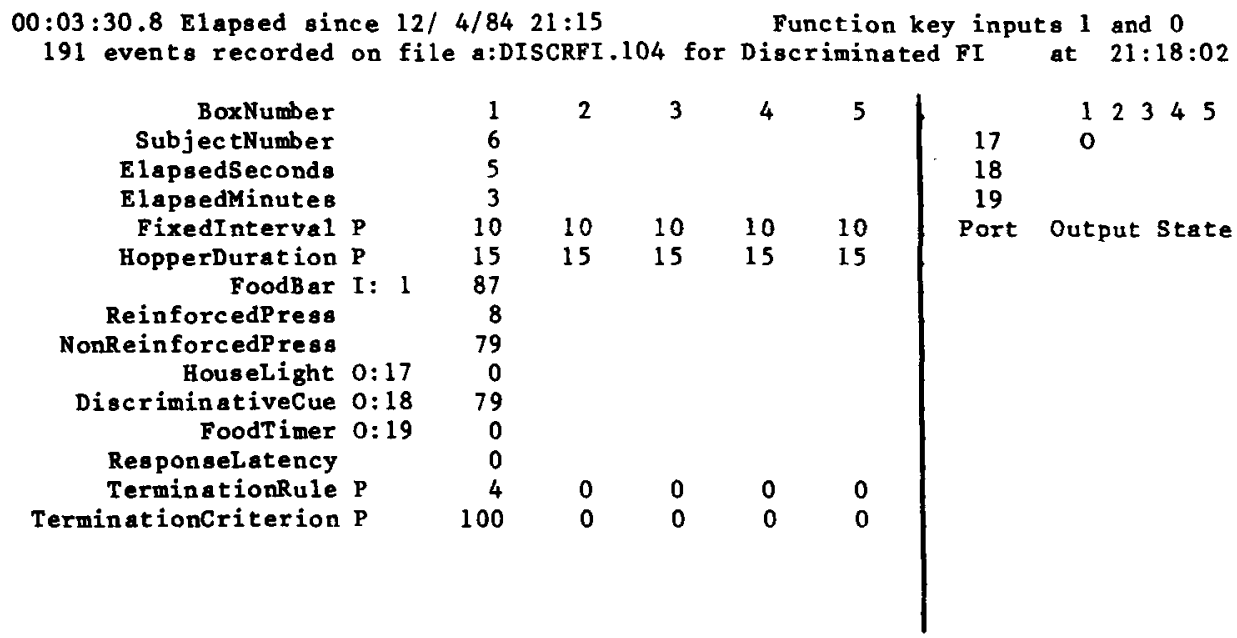

Next command: SUBJECT 1

Box 1, Subject 1, 13:22:28+ 0:03:02, records 1-191, print incomplete

Figure 1. A typical monitor screen display during execution of the DISCRFI paradigm, running with a keyboard simulation of barpresses. Session date and time information appear at the top of the screen. Two of the experimenter-controllable parameters, FixedInterval (in seconds) and HopperDuration (in tenths of a second), still have their default values; the experimenter has reset the other two, TerminationRule and TerminationCriterion, to request that the session stop after a 10-sec latency to respond after the cue onset. One subject is active in Box 1 , and reinforcement will be available after another $7.9 \mathrm{sec}$ (DiscriminativeCue counts down every $100 \mathrm{msec}$, and when it reaches 0 , the tone comes on). So far there have been 87 barpresses, of which 8 were reinforced. At the right side of the screen, the output display shows only Output 17, the houselight, as being active at that moment. At the bottom of the screen, the operator has asked for a summary of Subject 1's history in this session; the response shows that, as of elapsed time 3:02, there had been 191 records written to disk, and that the printing of data (as cumulative records) was not complete. This screen display is updated as often as practical, given the higher priority of the real-time control and the need to share the remaining CPU time with the cumulative-record printing routine.

[display] HELP [listing of all user commands]

HOWBUSY number-of-seconds [display mean and peak CPU utilization]

[for simulations,] EXERCISE specified-input-bit EVERY [average]

number-of-ticks OF/UNLESS specified-output-bit [is active] NOEXERCISE

KEYBOARDTEST specified-input-bit specified-input-bit

STOP [real-time operations, return to DOS]

The HOWBUSY, EXERCISE, and KEYBOARDTEST commands are included to facilitate testing of new paradigms. HOWBUSY reports CPU utilization (the mean and maximum CPU time occupied by the interrupt handler) and event generation (the increase in the backlog of unwritten event records still in the memory buffers) over the course of several seconds, under normal or simulated operation. EXERCISE simultaneously simulates barpresses on all bars, and can be used in conjunction with HOWBUSY to estimate peak loading on the system. The KEYBOARDTEST command allows the operator to disable the external interface and use the 10 function keys at the left of the keyboard to emulate two inputs each for the first five boxes. The screen is redrawn with only these five boxes' data, and a separate window displays emulations of all of the output bits defined in the dictionary. Figure 1 shows a display screen from the discriminated FI experiment during such keyboard emulation.
Production of cumulative records, such as the example shown in Figure 2, occurs automatically during real-time operation. The parameters of the graphs, such as the events to be cumulated, to cause tick marks, and to be reported in annotations in the right margin, are defined by the programmer and stored in a separate dictionary. Once each minute, the background program inspects each subject's list of graph pages already written and record of elapsed time, and if any page's data are ready for graphing (even if the subject is still active), that page will be graphed. Although the pages that together make up one continuous graph may be separated in the printing, each page includes subject numbers, time axes, and other information sufficient for recollation. After the last page of a subject's graphs is output, a table listing the final values on selected counters is also printed.

\section{DEVELOPMENT PLANS}

Future plans for FREDL can be divided into several phases. Of most immediate urgency is completion of the programmer's development tools and their associated documentation. These will include the interactive dictionary construction program, programs to automate the propa- 

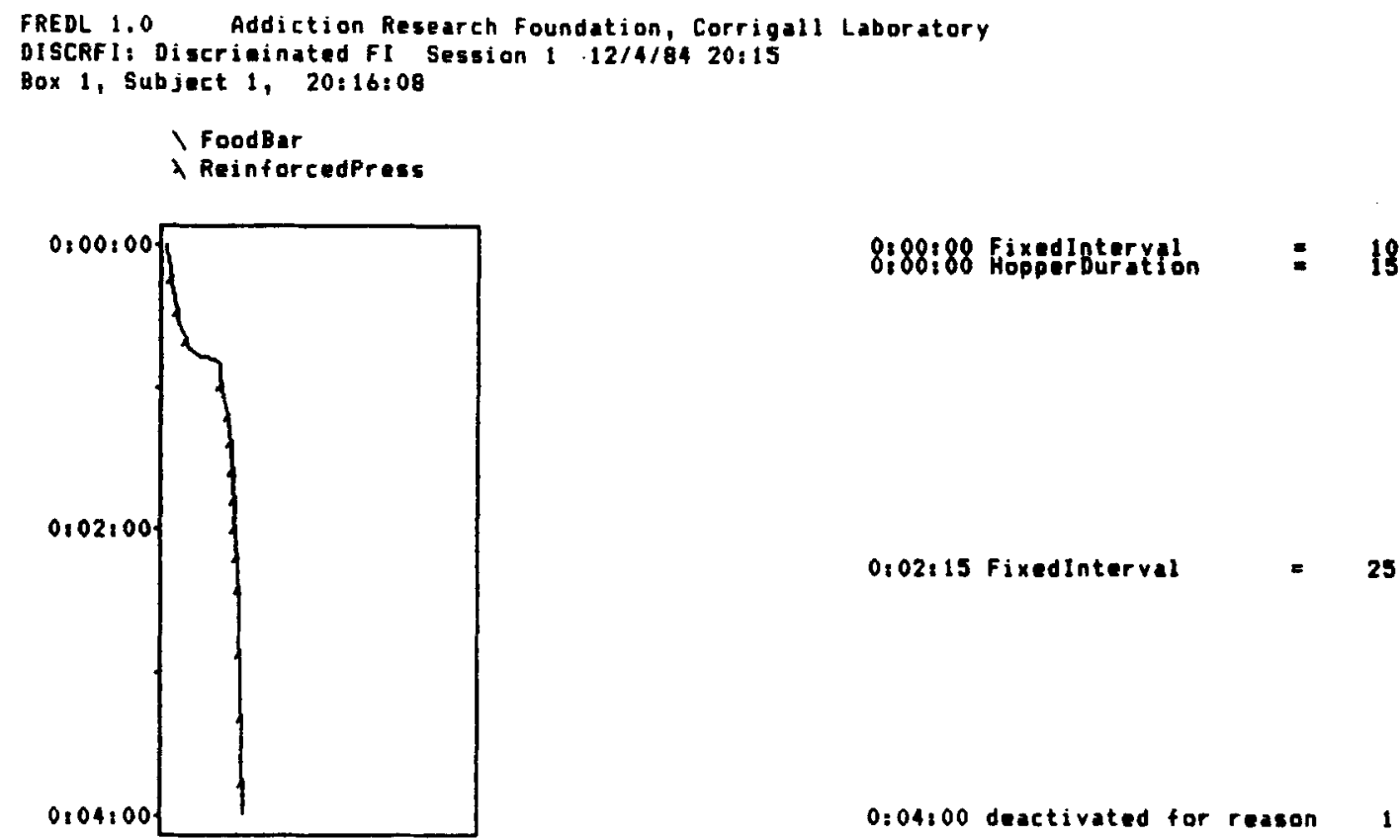

$0: 04: 00$ deactivated for reason 1
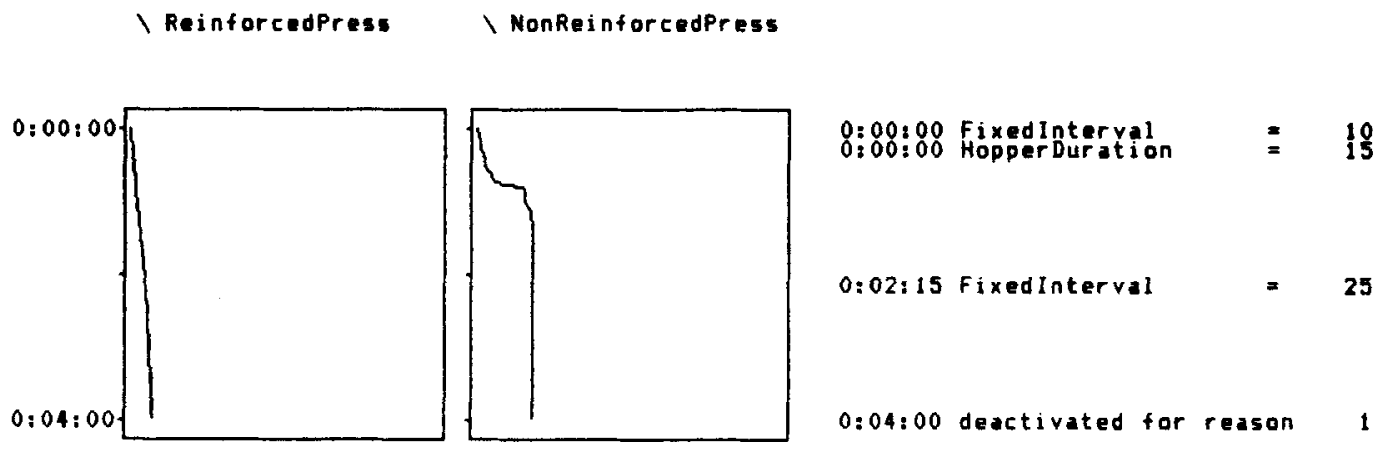

Figure 2. Cumulative records produced by the program on an Epson FX-100 printer, based on programmer-specified tables which control scale, events to be graphed, and other events to occur as annotations in the right margin. The upper graph is a traditional form, with total responses cumulated and reinforced ones shown as tick marks. The increase in interreinforcer interval occurring just after 2 min has elapsed is explained by the experimenter's increase of the fixed interval annotated in the right margin. The lower graph presents the same data in another scale and form, with reinforced and unreinforced responses cumulated separately. Pages of these records are printed as soon as their data are ready, even though their or other subjects continue to be active in the experiment.

gation of code and dictionary files from development to operation disks, and provision of a library of routines to facilitate off-line reading and analysis of the data stored on diskette.

The next stage will be the preparation of FREDL for export to other laboratories. This export will include adaptations for other printers and other reasonably compatible computer systems, more extensive documentation than is required internally, and instructions for constructing the hardware interfaces. We expect the program to be sold for a reasonable cost, rather than given away, but no decisions about price have yet been made.
When time becomes available, there are a number of possible improvements that could be made to FREDL. Currently, the number of user-defined counters is limited to 50, but a different memory-utilization strategy should allow that number to be increased substantially. To support such an increase, however, it will be necessary to rewrite some critical sections of the interrupt handler directly in machine code, to achieve the speed necessary to process more complex paradigms. Another planned improvement is to allow cumulative record abscissas to be labeled in either elapsed or real time, so that experiments on diurnal variation can be displayed more easily. 


\section{REFERENCES}

Emmett-OGlesby, M. W., Spencer, D. G., \& ARnoult, D. E. (1982) A TRS-80 based system for the control of behavioral experiments. Pharmacology, Biochemistry and Behavior, 17, 583-587.

KAPLAN, H. L. (1980). Applications of multiprogramming software to real-time experiments in psychology. Behavior Research Methods \& Instrumentation, 12, 137-151.

KAPLAN, H. L. (1982). An operating subsystem for continuous monitor- ing studies. Behavior Research Methods \& Instrumentation, 14, 146-159.

SNAPPER, A. G., KADdeN, R. M., \& INGuIs, G. B. (1982). State notation of behavioral procedures. Behavior Research Methods \& Instrumentation, 14, 329-342.

SPENCer, D. G., \& EMMETt-Oglesby, M. W. (1985). Parallel processing strategies in the application of microcomputers to the behavioral laboratory. Behavior Research Methods, Instruments, \& Computers, 17, 294-300. 